$\alpha$ (=mean free path/Planck mean free path) is independent of temperature. Such problems have previously been treated by several authors (e.g., King, Astrophys. J. 121, 711, 1955) in low orders of approximation, employing separate quadrature formulas in $\alpha$ and $\mu$ space $(\mu=\cos \theta)$. By using the single variable $\alpha \mu$ and uniformly spaced quadrature points, the characteristic equation for the problem can be solved with the aid of an approximating function which becomes exact as $N$ (the number of points) increases without limit. The limb darkening and the source function for the Milne problem, expressed in terms of the roots and constants of the characteristic equation, can then be evaluated exactly as explicit real integrals; in the grey case the results of Placzek and Mark are recovered. The present derivation does not employ complex variables. Because of the nature of the assumed absorption coefficient, the method is applicable to (e.g.) the picket-fence, Elsasser, and statistical (Mayer-Goody) models of line absorption, but not strictly to the Lyman-continuum blanketing effect. Numerical results for a number of cases are exhibited.

$\lambda 4430$ Band and Small Interstellar Particles. R. Stoeckly, Princeton University Observatory.$O$ stars whose spectra indicate interaction with nearby clouds appear to have weaker $\lambda 4430$ bands than other stars with the same color excess. Interstellar $C a$ II line components with high negative radial velocity were used as an indicator for the presence of nearby clouds. Sixteen stars, including 12 in Orion and Cygnus, show the deficiency. Such stars evidently modify the $\lambda 4430$ absorbers in nearby matter. One explanation is that the band may be associated with small interstellar particles $(r<0.1 \mu)$ which contribute little to extinction in the visible region except at $\lambda 4430$ and which are selectively destroyed in the vicinity of $\mathrm{O}$ stars. The restrictions which this would impose on the particle size and distribution are not strong, but the small particles would have to be much more numerous than in the theoretical distribution proposed by van de Hulst (1946).

Excitation of Metal Lines in Flares and in Active Prominences. E. Tandberg-Hanssen, High Altitude Observatory.-The relative strengths of emission lines from hydrogen and helium in the spectra of limb flares and of active prominences (loops, surges) generally resemble each other to the point of being indistinguishable. The relative intensities of metal lines seem, however, often to be different in flares and in prominences. In prominences, certain ionized titanium lines are stronger than lines of ionized iron, whereas the reverse holds true in flares. Other typical "prominence lines" come from $S c$ II and $Y$ II. Lines of ionized chromium are not especially strong in prominences relative to flares and may follow $F e$ II more closely. It looks as if "prominence lines" belong to elements of the first subgroups in the periodic system.

As a new classification criterion to distinguish between limb flares and active prominences we introduce the intensity ratio $M=I(F e \mathrm{II}, 4584) / I(T i$ II, 4572), and define an object a flare if $M \geqq 1$ and an active prominence if $M<1$. The selective excitation of these lines in flares and prominences seems mainly to be governed by the density differences, and the $M$ criterion is therefore related to the difference in density, not temperature, in flares and prominences.

\section{OB Spectral Group Classifications as Distance} Indicators. A. R. Upgren, Sproul Observatory, Swarthmore College.-In a paper presented by A. Beer at the last meeting of the American Astronomical Society at Cambridge (Astron. J. 67, 268, 1962 ), it was shown that the $R$ values derived from photoelectric narrow-band measurements of $\mathrm{Hr}_{\gamma}$ in early-type stars provide a rapid method of luminosity determination. The 434 stars examined are mostly brighter than the eighth magnitude.

The aim of this paper is to investigate the possibility of a close correlation between these $R$ values and the OB classifications of Slettebak and Stock (Z. Astrophys. 42,67, 1957) which extend down to a limiting photographic magnitude of about 13 . They divide early-type stars into three main groups known as $\mathrm{OB}^{+}, \mathrm{OB}$, and $\mathrm{OB}^{-}$, using objective prism plates of the Hamburg Schmidt telescope. If such a correlation exists for those brighter stars which are at present common to both systems, then it should also hold for stars between magnitudes 8 and 13.

In order to test this correlation, only stars can be used in which $\mathrm{H}_{\gamma}$ is free of emission. Such emission stars have been eliminated from consideration. Altogether 30 stars from Beer's list have available OB spectral types. These yield the following results: for $\mathrm{OB}^{+}, n=5$ and $\bar{R}=1.037 \pm 0.010$; for $\mathrm{OB}, n=15$ and $\bar{R}=1.063 \pm 0.008$; and for $\mathrm{OB}^{-}$, $n=10$ and $\bar{R}=1.126 \pm 0.006$, where $n$ and $\bar{R}$ are the number of stars and the mean $R$ values, respectively, for each spectral group. The calibration curve of $R$, i.e., the corresponding absolute magnitudes, derived from 130 standard stars as reproduced in Beer's more detailed paper (Monthly Notices Roy. Astron. Soc., to be published) gives: $-5.9 \pm 0.6,-4.6 \pm 0.3$ and $-2.5 \pm 0.2$, respectively, for the three $R$ values quoted above.

In view of the intrinsic accuracy of this calibration of $R$ vs $M$, the assignment of these luminosi- 